\title{
A Model for COVID-19 Prediction in Iran Based on China Parameters
}

\author{
Bushra Zareie, PhD Student ${ }^{1,2}$; Amin Roshani, PhD ${ }^{3}$; Mohammad Ali Mansournia, MD, MPH, PhD ${ }^{4}$; Mohammad Aziz Rasouli, MSc ${ }^{2,5}$; \\ Ghobad Moradi, MD, MPH, PhD ${ }^{2 *}$ \\ 'Department of Epidemiology, School of Public Health, Hamadan University of Medical Sciences, Hamadan, Iran \\ ${ }^{2}$ Social Determinants of Health Research Center, Research Institute for Health Development, Kurdistan University of Medical Sciences, \\ Sanandaj, Iran \\ 3epartment of Statistics, Razi University, Kermanshah, Iran \\ ${ }^{4}$ Department of Epidemiology and Biostatistics, School of Public Health, Tehran University of Medical Sciences, Tehran, Iran \\ ${ }^{5}$ Clinical Research Development Center, Kowsar Hospital, Kurdistan University of Medical Sciences, Sanandaj, Iran
}

\begin{abstract}
Background: The rapid spread of COVID-19 virus from China to other countries and outbreaks of disease require an epidemiological analysis of the disease in the shortest time and an increased awareness of effective interventions. The purpose of this study was to estimate the COVID-19 epidemic in Iran based on the SIR model. The results of the analysis of the epidemiological data of Iran from January 22 to March 24, 2020 were investigated and prediction was made until April 15, 2020.

Methods: By estimating the three parameters of time-dependent transmission rate, time-dependent recovery rate, and timedependent death rate from Covid-19 outbreak in China, and using the number of Covid-19 infections in Iran, we predicted the number of patients for the next month in Iran. Each of these parameters was estimated using GAM models. All analyses were conducted in R software using the mgcv package.

Results: Based on our predictions of Iran about 29000 people will be infected from March 25 to April 15, 2020. On average, 1292 people with COVID-19 are expected to be infected daily in Iran. The epidemic peaks within 3 days (March 25 to March 27, 2020) and reaches its highest point on March 25, 2020 with 1715 infected cases.

Conclusion: The most important point is to emphasize the timing of the epidemic peak, hospital readiness, government measures and public readiness to reduce social contact.

Keywords: China, COVID-19, Iran, Prediction

Cite this article as: Zareie B, Roshani A, Mansournia MA, Rasouli MA, Moradi G. A Model for COVID-19 Prediction in Iran Based on China Parameters. Arch Iran Med. 2020;23(4):244-248. doi: 10.34172/aim.2020.05.
\end{abstract}

Received: March 16, 2020, Accepted: March 21, 2020, ePublished: April 1, 2020

\section{Introduction}

Transmission of coronavirus-associated pneumonia 2019 (COVID-19) began in Wuhan, China on December 31, 2019. ${ }^{1}$ According to the latest World Health Organization (WHO) report on March 11, 2020, there were 118329 confirmed cases and 4627 deaths worldwide, with 8042 confirmed cases and 291 deaths reported in Iran. The WHO named it a global pandemic on March 11, 2020 because of the rapid outbreak of the disease worldwide. ${ }^{3}$ The course of each epidemic depends on a number of important factors such as the basic reproductive rate, the doubling time or serial interval, and the fatality rate. Estimating the epidemiological factors of COVID-19 and modeling-based prediction are useful in assessing epidemic transmission rates, predicting epidemic trends, and designing control measures. ${ }^{4,5}$

The rapid spread of COVID-19 virus from China to other countries and outbreaks of disease require an epidemiological analysis of the disease in the shortest time and increased awareness of effective interventions. What has happened in China shows that quarantine, social distancing, and isolation of infected populations can contain the epidemic. This impact of the COVID-19 response in China is encouraging for many countries where COVID-19 has begun to expand. However, it is not clear whether other countries can implement the measures that the Chinese eventually adopted to reduce and control the incidence in the future. ${ }^{4,6}$

There are various models for predicting the spread of viruses. Along with vaccines or diagnostic tests, mathematical modeling can be a useful tool for designing strategies for prediction and designing appropriate interventions for rapid control of infectious diseases, if not treated effectively. The purpose of this study was to estimate the COVID-19 epidemic in Iran based on the SIR model. The results of the analysis of the epidemiological data of Iran from January 22 to March 24, 2020 were investigated and the prediction was made until April 15, 2020. 


\section{Materials and Methods}

A simple model of SARS and the precise prediction by Zhang et $\mathrm{al}^{7}$ motivated us to use this model for COVID19. Limited laboratory kits, prolonged test results, people's lack of awareness and non-referral to medical centers led to incomplete registration of infected, suspected or dead cases in the onset days of the epidemic in Iran. In this situation, it is better to use a model with few parameters and include time-dependent rates. Suppose equation 1 includes four parameters: $\beta(\mathrm{t})$ time-dependent transmission rate, $\mathrm{Y}(\mathrm{t})$ time-dependent recovery rate, $\mu(t)$ time-dependent death rate, and $\mathrm{I}(\mathrm{t})$ time-dependent infected number. The number of patients in our model is a function of time. We predict the number of patients day by day. Therefore, we use the second equation that is based on the first equation.

$$
\begin{array}{lll} 
& d I(t) / d t=\beta(\mathrm{t}) I(t)-(\Upsilon(\mathrm{t})+\mu(\mathrm{t})) I(t), & I\left(t_{0}\right)=I_{0} \\
\text { Equation(1) } & I(t+1)=I(t)+\beta(\mathrm{t}) I(t)-(\Upsilon(\mathrm{t})+\mu(\mathrm{t})) I(t), & I\left(t_{0}\right)=I_{0} \\
\text { Equation(2) } &
\end{array}
$$

Each parameter is defined as follows:

$$
\beta(t)=\frac{\text { the number of daily diagnosesd covid19 patients at time } \mathrm{t}}{\text { the number of accumulated covid19 patients at time } \mathrm{t}}
$$

$$
Y(t)=\frac{\text { the number of daily recovered covid19 patients at time } \mathrm{t}}{\text { the number of accumulated covid19 patients at time } \mathrm{t}}
$$

$$
\mu(t)=\frac{\text { the number of daily died covid19 patients at time } \mathrm{t}}{\text { the number of accumulated covid19 patients at time } \mathrm{t}}
$$

We fit the model in three steps.

Step 1: In the first step, we analyzed the Chinese data during the outbreak of covid19 and obtained the Chinese model. This allowed us to estimate parameters by accessing more data (Figure 1A-D).

Step 2: We put the fitting function for $\beta(t), U(t), m(t)$ in the first step along with the actual trend parameters of Iran and found that the behavior parameters of Iran are similar to China (Figure 2A-C).

Step 3: The conclusion of step 2 has many benefits for us because we cannot obtain the behavior of the parameters for Iran in a short time. Accordingly, we can use the Chinese parameters to predict the number of Iranian patients in the last step (Figure 3).

But the question remains on how to fit each parameter in China model. Many data in the environmental sciences do not fit simple linear models and are best described by "wiggly models", also known as generalized additive models (GAMs). We used GAMs to estimate $\beta(\mathrm{t}), \mathrm{U}$ $(\mathrm{t}), \mathrm{m}(\mathrm{t}), \mathrm{I}(\mathrm{t})$ and models fit by Restricted Maximum likelihood (REML).

One of the main reasons for using GAMs models was that we did not know the distributions of parameters; so, we could not use a parametric approach like non-linear least squares.
We performed all analyses using the R software. Also, the mgcr package in $\mathrm{R}$ was used to fit GAMs.

\section{Results}

The first phase (March 25, 2020 - March 27, 2020): On average, we expect daily diagnoses more than 16000 people until the end of the initial phase.

The second phase (March 28, 2020 - April 15, 2020): In this phase, it is expected that the downward trend will continue until April 15, 2020 if the intervention measures continue at current intensity.

Assuming that the epidemic continues to develop from March 25, 2020 to April 15, 2020, a total of 28425 people are expected to be diagnosed with COVID-19 across the country. In other words, on average, 1292 people with COVID-19 are expected to be infected daily in Iran. The epidemic peaks within one week (March 25, 2020 to March 27, 2020) and reaches its highest point on March 25, 2020 with 1715 infected cases (Figure 3; Table 1).

\section{Discussion}

Our estimation shows that the COVID-19 epidemic trend in Iran will rise from March 25, 2020 and will peak during March 25, 2020 - March 27, 2020, and there will be a decreasing trend from March 28, 2020 - April 15, 2020. Under such circumstances, two types of hypotheses can be considered for the adequacy of the current measures and whether the epidemic peak will occur during March 15-21, 2020. Assuming that the current measures are inefficient and inadequate, the number of estimated cases will continue to increase by the end of March. On the other hand, the current control measures are effective and sufficient and the number of cases estimated after the epidemic peak will go down. This model may be close to reality if intervention measures continue at the current intensity. ${ }^{8}$ Assuming the prevention and control measures be sufficient and effective in Iran behave like China, to depict two different levels of effect of the measures in reducing transmission probability.

At the time of the outbreak of COVID-19, the best and most urgent steps must be taken to overcome the coronavirus epidemic. The fight against coronavirus infection should be an emergency. It can only be overcome with active cooperation of various businesses and professions such as the medical industry, transportation, government, manufacturers of technology products, etc. ${ }^{9}$

Several essential tasks must be done while controlling the outbreak; 1) Controlling the virus source. In the face of the virus, the most effective strategy is to keep any suspected infected patient in a relatively confined space (hospital or home) to prevent the virus from spreading. Verified patients should be treated at the hospital immediately. 2) Controlling the virus spread. Restrictive measures must be taken against the general population or the population that may spread the disease to prevent a major epidemic 

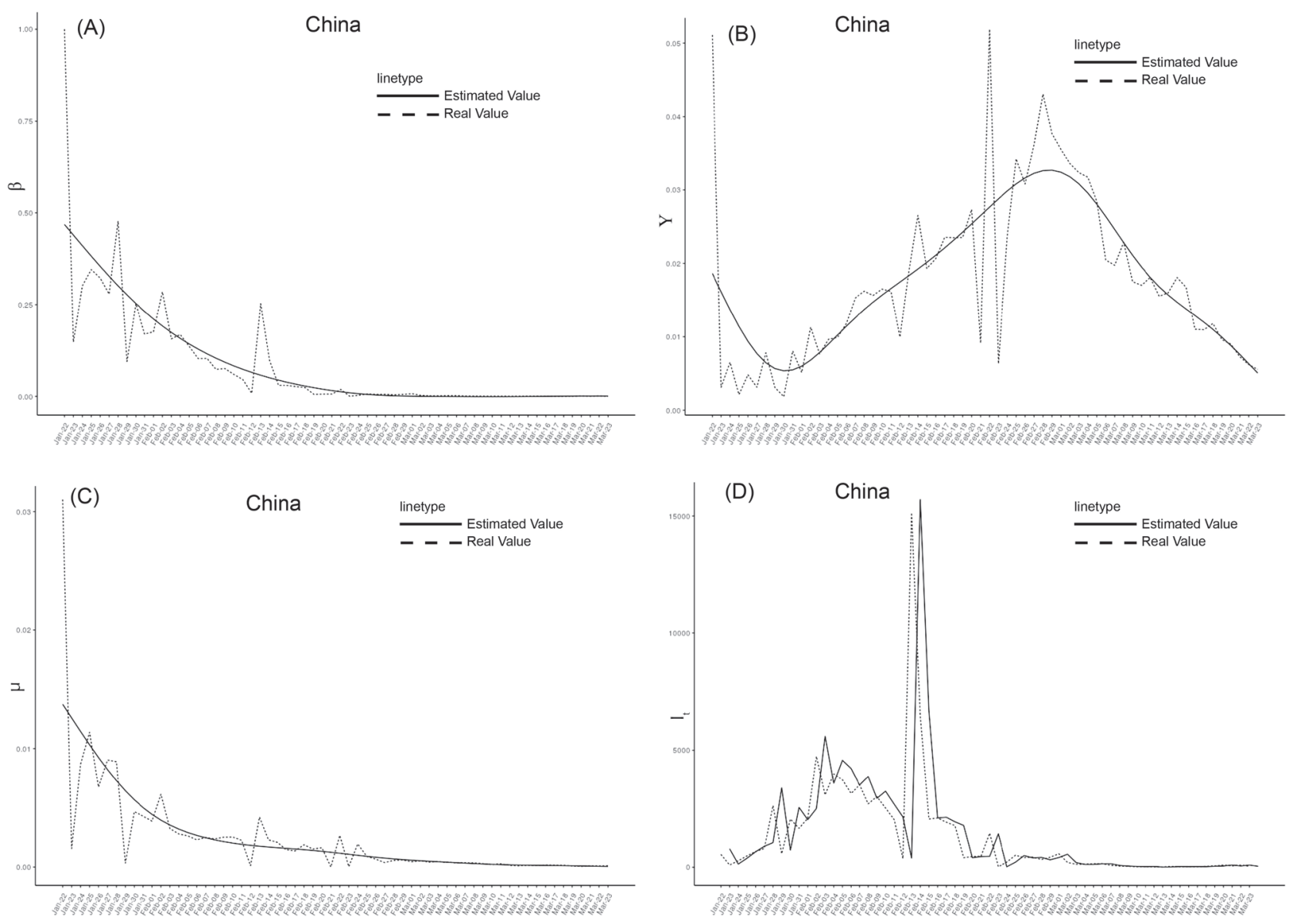

Figure 1. (A) Time-Dependent Transmission Rate People in China from January 22, 2020 to March 23, 2020. (B) Time-Dependent Recovery Rate People in China from January 22, 2020 to March 23, 2020. (C) Time-Dependent Death Rate People in China from January 22, 2020 to March 23, 2020. (D) Number of Infected People in China from January 22, 2020 to March 23, 2020.

from continuing. 3) Virus tracking. In addition, the main source of the virus must be traced to understand the origin of the virus and effective measures must be taken to completely eliminate the source of the virus. ${ }^{9-11}$

For an entire contaminated or potentially contaminated city, continuous air detection must be carried out to effectively detect or trace the virus in the air, and masks must be worn by all citizens, especially in public places. New mobile hospitals should be built that can handle suspected cases in a centralized manner. This is essential to alleviating the pressure of treating the masses of infected patients in large hospitals. For example, during this outbreak, the Huoshenshan Hospital in Wuhan was built in only 10 days for people infected with the coronavirus. During the whole process, the epidemic situation should be reported in a timely and transparent manner. It also prevents unexpected public pressure and reduces unnecessary mental health problems.

Artificial intelligence technology can play a key role in almost every aspect, including traffic management, infection detection, logistics supply chain, etc. This is a very important feature of a modern data-driven smart city. If the status of each citizen is listed, all can be accurately tracked and any population can be accommodated. Therefore, the flow of population can be controlled in a more orderly manner. Artificial intelligence technologies can be used to employ smart devices to support diagnosis and treatment, and can be used in telecommunications, online training and intelligent manufacturing to ensure minimal disruption to people's lives. Some hospitals use smart systems. Train stations can install powerful thermal imagers to measure the passengers' body temperature. During the whole control process, efficiency and speed is extremely important and cross-disciplinary research should be conducted..$^{9-11}$

In response to the outbreak, China expanded its dayto-day contact with the $\mathrm{WHO}$, regardless of economic disadvantages, and developed comprehensive multifaceted approaches to tackle the virus and prevent further exposure to the virus by taking rigorous and unprecedented measures arising from a sense of responsibility to citizens. It also disseminated its epidemic information openly, transparently, responsibly and in a timely manner to all other countries and international organizations. ${ }^{8}$

There are difficult decisions for governments to make. How people respond to recommendations on preventing 

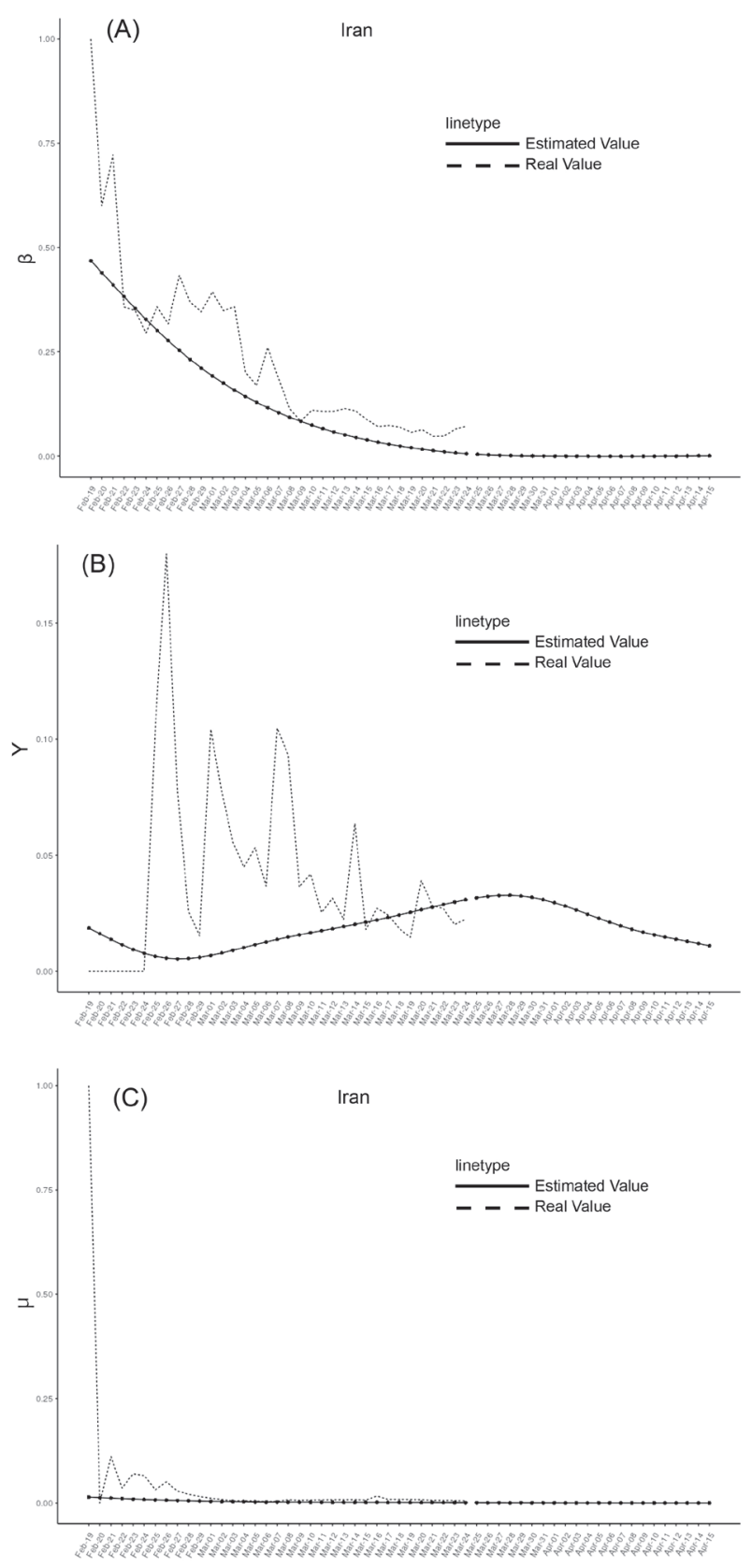

Figure 2. (A) Time-Dependent Transmission Rate People in Iran with $\beta(\mathrm{t})$ Estimated of China. (B) Time-Dependent Recovery Rate People in Iran with $\mathrm{Y}(\mathrm{t})$ Estimated of China. (C) Time-Dependent Death Rate People in Iran with $\mu(t)$ Estimated of China.

transmission is more important than government measures. The government's strategy of communicating with the public to inform them about infection prevention is also crucial. Extra support for managing the recession is also important. However, it is not clear whether other countries can implement the measures that the Chinese eventually adopted to reduce and control the incidence in the future.

Other countries can adopt only certain elements of China's strategy, including suspension of public transportation, closure of recreational areas and a ban on public assemblies that have been the most effective

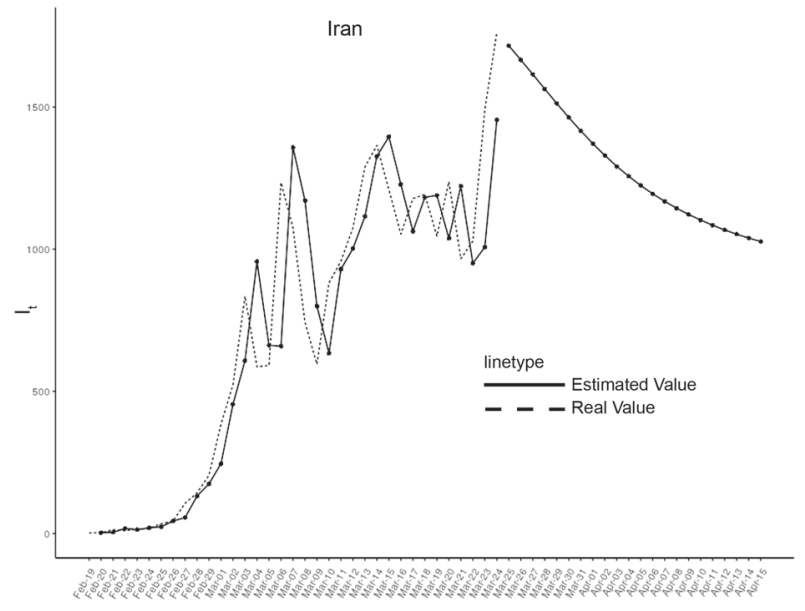

Figure 3. Predicted Number of Infected People in Iran from March 25, 2020 to April 15, 2020.

Table 1. Number of Infected People in Iran from March 25, 2020 to April 15,2020

\begin{tabular}{llll}
\hline Date & $\mathbf{I}(\mathbf{t})$ & Date & $\mathbf{I}(\mathbf{t})$ \\
\hline 3.25 .2020 & 1715 & 4.5 .2020 & 1224 \\
\hline 3.26 .2020 & 1666 & 4.6 .2020 & 1195 \\
\hline 3.27 .2020 & 1615 & 4.7 .2020 & 1168 \\
\hline 3.28 .2020 & 1563 & 4.8 .2020 & 1144 \\
\hline 3.29 .2020 & 1513 & 4.9 .2020 & 1122 \\
\hline 3.30 .2020 & 1463 & 4.10 .2020 & 1102 \\
\hline 3.31 .2020 & 1416 & 4.11 .2020 & 1084 \\
\hline 4.1 .2020 & 1371 & 4.12 .2020 & 1068 \\
\hline 4.2 .2020 & 1330 & 4.13 .2020 & 1053 \\
\hline 4.3 .2020 & 1291 & 4.14 .2020 & 1039 \\
4.4 .2020 & 1256 & 4.15 .2020 & 1027 \\
\hline
\end{tabular}

measures in curbing the epidemic in China. Hospitals can increase their storage of medical equipment and number of beds, and the government can emphasize the importance of hand washing and staying home if ill. ${ }^{12}$

Extensive capacity is needed for such a level of medical care, which is a health emergency and is particularly challenging. We hope that the analytical results of this study will help to clarify important aspects of the outbreak so that the spread of coronavirus in different locations can be minimized in the shortest time possible.

One of the limitations of this study is the uncertainty of data on the first days of the epidemic in Iran. The distribution of data in the first week of the epidemic was such that the fitting of each parameter of the model with Iranian data through non-linear least squares or polynomial regression models posed problems so that we could not identify a better model with AIC or BIC criteria

In conclusion, the most important point is to emphasize the timing of the epidemic peak, hospital readiness, government measures and public readiness to reduce social contact. 


\section{Authors' Contribution}

Data analysis was led by BZ and AR who programmed the model with help from GM and MAM who planned the inference framework. Data was collected from online sources. MAR, BZ, GM and MAM wrote the paper. All authors interpreted the findings, contributed to writing the manuscript, and approved the final version for publication.

\section{Conflict of Interest Disclosures}

The authors declare that they have no competing interests.

\section{Ethical Statement}

Ethical issues of this study have been approved by ethics committee of Kurdistan University of Medical Sciences.

\section{Acknowledgements}

This study was funded by Vice Chancellor for Research and Technology of Kurdistan University of Medical Sciences, Sanandaj, Iran.

\section{References}

1. Zhan C, Tse C, Fu Y, Lai Z, Zhang H. Modelling and Prediction of the 2019 Coronavirus Disease Spreading in China Incorporating Human Migration Data. medRxiv 2020. doi:10.1101/2020.02.18.20024570

2. Report-51.WCdC-s. Available from: https://www.who.int/docs/ default-source/coronaviruse/situation-reports/20200311sitrep-51-covid-19.pdf?sfvrsn=1ba62e57_4. Published March 11, 2020).

3. Novel Coronavirus Pneumonia Emergency Response Epidemiology Team. The epidemiological characteristics of an outbreak of 2019 novel coronavirus diseases (COVID-19) in China. Zhonghua Liu Xing Bing Xue Za Zhi. 2020;41(2):145151.

4. Anderson RM, Heesterbeek $\mathrm{H}$, Klinkenberg D, Hollingsworth TD. How will country-based mitigation measures influence the course of the COVID-19 epidemic? Lancet. 2020; 395(10228):931-4. doi: 10.1016/S0140-6736(20)30567-5

5. Zou L, Ruan F, Huang M, Liang L, Huang H, Hong Z, et al. SARS-CoV-2 viral load in upper respiratory specimens of infected patients. N Engl J Med. 2020;382:1177-9. doi: 10.1056/NEJMc2001737

6. Coronavirus disease 2019 (COVID-19) Situation Report - 44. Avaialable from: https://www.who.int/docs/default-source/ coronaviruse/situation-reports/20200304-sitrep-44-covid-19. pdf?sfvrsn=783b4c9d_2. Accessed March 5, 2020.

7. Zhang J, Li J, Ma Z. Global dynamics of an SEIR epidemic model with immigration of different compartments. Acta Mathematica Scientia. 2006;26(3):551-67. doi: 10.1016/ S0252-9602(06)60081-7

8. Wang H, Wang Z, Dong Y, Chang R, Xu C, Yu X, et al. Phaseadjusted estimation of the number of Coronavirus Disease 2019 cases in Wuhan, China. Cell Discov. 2020;6(1):1-8. doi: 10.1038/s41421-020-0148-0

9. Xu C, Luo X, Yu C, Cao SJ. The 2019-nCoV epidemic control strategies and future challenges of building healthy smart cities. Indoor and Built Environment. London, England: SAGE Publications; 2020.

10. She J, Jiang J, Ye L, Hu L, Bai C, Song Y. 2019 novel coronavirus of pneumonia in Wuhan, China: emerging attack and management strategies. Clin Trans Med. 2020;9(1):1-7. doi:10.1186/s40169-020-00271-z

11. Wu JT, Leung K, Leung GM. Nowcasting and forecasting the potential domestic and international spread of the 2019nCoV outbreak originating in Wuhan, China: a modelling study. Lancet. 2020;395(10225):689-97. doi: 10.1016/S01406736(20)30260-9

12. Cohen J KK. Strategies shift as coronavirus pandemic looms. Science. 2020;367(6481):962-3. doi: 10.1126/ science.367.6481.962. 